\title{
Superimposed XOR: A New Physical Layer Network Coding Scheme for Two-Way Relay Channels
}

\author{
Jianquan $\mathrm{Liu}^{\dagger}$, Meixia $\mathrm{Tao}^{\dagger}$, Youyun $\mathrm{Xu}^{\dagger *}$, and Xiaodong Wang ${ }^{\ddagger}$ \\ ${ }^{\dagger}$ Dept. of Electronic Engineering, Shanghai Jiao Tong University, Shanghai, 200240, China \\ *PLA University of Science and Technology, Nanjing 210007, China \\ $\ddagger$ Dept. of Electrical Engineering, Columbia University, New York, NY 10027, USA \\ Emails: \{jianquanliu, mxtao, xuyouyun\}@sjtu.edu.cn, wangx@ee.columbia.edu
}

\begin{abstract}
We present a new physical layer network coding (PLNC) scheme, called superimposed XOR, for two-way relay channels. The new scheme specifically takes into account the channel as well as information asymmetry in the broadcast phase of two-way relaying. It is based upon both bitwise XOR and symbol-level superposition coding. We first derive its achievable rate regions when integrated with two known time control protocols over Gaussian channels. We then demonstrate its average maximum sum-rate and service delay performance over fading channels. Compared with the existing bitwise XOR and symbol-level superposition coding, the proposed superimposed XOR scheme achieves larger rate region in asymmetric channels. As a result, it performs much better in terms of averaged maximum sum-rate and service delay over fading channels. Numerical results also show that the proposed practical PLNC closely approaches the capacity bound given by the informationtheoretic random binning.
\end{abstract}

\section{INTRODUCTION}

Two-way relaying, where two source nodes exchange information with the help of a relay node, has recently gained a lot of research interests [1]-[7]. It is shown able to overcome the half-duplex constraint and significantly improve the system spectral efficiency in relay-based cooperative networks. Upon receiving the bidirectional information flows, the relay node combines them together and then broadcasts to the two desired destinations. The operation at the relay resembles network coding, a technique originally developped for wireline networks. It is thus often referred to as physical layer network coding (PLNC) [5] or analog network coding (ANC) [6].

Researchers have attempted to find the best achievable rate region of two-way relay channels [8]-[10]. Oechtering, et al. obtained the capacity region of the broadcast phase in terms of the maximal probability of error [8]. The achievable rate region of a two-way relay channel considering both the multiple-access phase (i.e. the two source nodes transmit simultaneously to the relay node) and the broadcast phase was studied by Xie using random binning [9]. Kim, et al. [10], [11] further broadened the frontier of the achievable rate region by

This work is supported by Shanghai Educational Development Foundation (No. 2008CG14) and by Natural Science Foundation of Shanghai (No. 08ZR1410400). allowing optimal time share between different transmission phases. Since the achievability of the capacity and lower bound in [8]-[10] are based on random coding together with typical set decoding at the broadcast phase, we denote all these schemes as random binning ${ }^{1}$ for convenience. Note that these results all assumed full decoding at the relay. The capacity region of two-way relaying with partial decoding is still an open topic.

Meanwhile, a number of practical PLNC schemes for two-way relay Gaussian channels have also been proposed and analyzed, such as bit-level XOR [12], [13], symbollevel superposition coding [7], [14] and amplify-and-forward (AF) [5], [7]. In particular, authors in [7] obtained the rate pair expressions for superposition based relaying. Authors in [12] analyzed the maximum achievable sum-rate for XOR based relaying with optimal time control. It is worth mentioning that the asymmetry in both packet size and channel gain of the two transmitting nodes are considered in [12]. Oechtering also studied the optimal time control for superposition scheme [14]. Despite all these attempts, we observe that there is still a large gap between the rate regions that can be achieved by the practical PLNC schemes and the optimal random binning in asymmetric channels.

In this paper, we study practical and capacity approaching PLNC schemes over two-way relay channels. In this regard, we propose a novel PLNC scheme, named as superimposed $X O R$, tailored for asymmetric broadcast channels. Combining it with two known transmission protocols: 4-step with direct link [10] and 2-step with no direct link, we analyze its achievable rate regions over Gaussian channels. We then illustrate the system average maximum sum-rate and service delay in fading channels using numerical results. For comparison, the traditional XOR and superposition are also considered. Results show that the performance of the proposed superimposed XOR-based PLNC scheme outperforms these schemes. It also closely approaches the best performance that is achieved by random binning, in the high SNR regime.

\footnotetext{
${ }^{1}$ The random binning used throughout this paper refers to the technique to combine the two independent messages from the two source nodes at the relay for broadcasting.
} 


\section{System MODEL}

\section{A. Channel model}

We consider a two-way relay channel which consists of two source nodes and one relay node. The source nodes, denoted as 0 and 2, wish to exchange information with the help of the relay node, denoted as 1 . We assume that all the nodes operate in the half-duplex mode. The channel on each communication link is assumed to be corrupted with Rayleigh fading and additive white Gaussian noise. The instantaneous signal-to-noise ratio (SNR) of the link from node $i$ to node $j$ is denoted as $\gamma_{i j}$, for $i, j \in\{0,1,2\}$, and it counts both channel gain and transmit power. Note that $\gamma_{i j}$ may not be equal to $\gamma_{j i}$ as the channels are considered here may not be reciprocal. The channel capacity in bit/s/Hz of the link from node $i$ to node $j$ is determined by the SNR on the link and denoted as $C_{i j} \triangleq C\left(\gamma_{i j}\right)=\log _{2}\left(1+\gamma_{i j}\right)$. Throughout this paper, we assume that the relay needs to fully decode the information of the two source nodes. The sum-rate capacity of the multiple access channel (MAC) when nodes 0 and 2 are transmitting simultaneously to node 1 is denoted as $C_{\mathrm{m}} \triangleq C\left(\gamma_{01}+\gamma_{21}\right)=\log _{2}\left(1+\gamma_{01}+\gamma_{21}\right)$.

\section{B. Time control protocols}

Two-way relaying involves not only PLNC at the relay node but also time control for node transmission. In this subsection, we review some existing time control protocols. Similar to [12], we name the protocols based on the number of time steps to finish one round of information exchange between the source nodes. We focus on the 4-step and 2-step protocols in this paper.

1) 4-step protocol: In the first step, node 0 transmits for $\lambda_{1}$ time duration and node 1 and 2 listen. In the second step, node 2 transmits for $\lambda_{2}$ time duration and node 1 and 0 listen. In the third step, nodes 0 and 2 transmit simultaneously for $\lambda_{3}$ time duration while node 1 listens. In the fourth step, node 1 transmits for $\lambda_{4}$ time duration and node 0 and 2 listen. Without loss of generality, the total time duration is normalized to one, i.e., $\sum_{i=1}^{4} \lambda_{i}=1$.

2) 2-step protocol: In 2-step protocol, nodes 0 and 2 first transmit simultaneously for $\lambda_{1}$ time duration while node 1 listens. Next, node 1 transmits for $\lambda_{2}$ time duration and nodes 0 and 2 listen. This protocol can be regarded as a special case of the 4-step protocol by letting $\lambda_{1}=\lambda_{2}=0$. No direct link is exploited here.

\section{Proposed PLNC SCHEME}

Upon decoding the two bit sequences $\mathbf{b}_{0}$ and $\mathbf{b}_{2}$ from the source nodes to be exchanged, the relay node will perform PLNC on $\mathbf{b}_{0}$ and $\mathbf{b}_{2}$ and then broadcast to the two destinations. Before introducing the proposed PLNC scheme, we briefly discuss the bit-level XOR and symbol-level superposition coding which the proposed scheme is based upon.

\section{A. Bit-level XOR}

The relay node performs bitwise XOR on the two bit sequences as $\mathbf{b}_{0} \oplus \mathbf{b}_{2}$. In the case where the lengths of the two sequences are not equal, there are two methods to perform the XOR. The first one is to pad the short sequence with zero bits so as to make it having the same length as the long sequence and then perform XOR. In the second method, the long sequence will be partitioned into two sub-sequences, with one having the same length as the short sequence. XOR is then performed on the short sequence and the sub-sequence with equal length and the resulting bit sequence is broadcasted to both receivers. The other sub-sequence will be transmitted alone to its desired receiver. In practice, which method to use depends on the relationship of the channel gains in the broadcast phase of two-way relaying [12].

\section{B. Symbol-level superposition}

The relay encodes the two bit sequences $\mathbf{b}_{0}$ and $\mathbf{b}_{2}$ separately into baseband signal sequences $\mathbf{x}_{0}$ and $\mathbf{x}_{2}$, and then superimposes them together as $\sqrt{\theta} \mathbf{x}_{0}+\sqrt{1-\theta} \mathbf{x}_{2}$. Here $\theta$ is a power allocation coefficient [7]. The signal $\sqrt{\theta} \mathbf{x}_{0}+\sqrt{1-\theta} \mathbf{x}_{2}$ is then broadcasted directly. Unlike bit-level XOR, there is no need to consider the issue of asymmetry in bit length.

\section{Proposed superimposed XOR}

As will be shown in Section IV-E of this paper, for symmetrical broadcast channels $\left(\gamma_{10}=\gamma_{12}\right)$, bit-level XOR is capacity-achieving whereas superposition coding is suboptimal. But for asymmetrical channels $\left(\gamma_{10} \neq \gamma_{12}\right)$, bit-level XOR becomes inferior to superposition at certain rate regions. The proposed superimposed XOR is specifically designed for asymmetrical broadcast channels and it utilizes the advantages of both XOR and superposition schemes. The details are as follows.

If the lengths of two bit sequences $\mathbf{b}_{0}$ and $\mathbf{b}_{2}$ are equal, then the conventional bitwise XOR is performed. Otherwise, there are two methods to process the two sequences. The first method is the same as the one in bit-level XOR scheme, that is, padding the short sequence with zero bits. In the second method, the relay node first partitions the long sequence, say $\mathbf{b}_{2}$, into two sub-sequences as $\mathbf{b}_{2}=\left[\mathbf{b}_{2}^{\prime} \mathbf{b}_{2}^{\prime \prime}\right]$, where the subsequence $\mathbf{b}_{2}^{\prime}$ has the same length as $\mathbf{b}_{0}$. It then encodes the XORed sub-sequence $\mathbf{b}_{0} \oplus \mathbf{b}_{2}^{\prime}$ and the sub-sequence $\mathbf{b}_{2}^{\prime \prime}$, separately. We denote the resulting coded symbol sequences as $\mathbf{x}_{0}$ and $\mathbf{x}_{2}$. Finally, the relay superimposes them together as $\sqrt{\theta} \mathbf{x}_{0}+\sqrt{1-\theta} \mathbf{x}_{2}$ which is broadcasted directly to the two destinations. Here, $\theta$ is also a power allocation coefficient, $\mathbf{x}_{0}$ is to be received by both destinations, and $x_{2}$ is to be received by one destination only. By changing the value of $\theta$, we can obtain different rate pairs in the broadcast phase. Which of the above two methods to use depends on the relationship between $\gamma_{10}$ and $\gamma_{12}$ in the broadcast channels. This shall be discussed in detail in the proof of the rate regions in the next section.

Note that Liu, et al. proposed a joint network coding and superposition coding (JNSC) scheme for information exchange among more than two users in a wireless relay network [15]. 
Therein, two XOR-ed packets generated by information from three nodes are superimposed. Our proposed superimposed XOR scheme differs from the JNSC [15] in that our scheme performs superposition on only one XOR-ed packet and the sub-packet obtained by partitioning the longer bit sequence.

For ease of writing, if the considered three PLNC schemes (XOR, superposition, and superimposed XOR) are combined with the 4-step time control protocol, we denote them as 4S-XOR, 4S-SUP and 4S-SuX, respectively. Likewise, when combined with 2-step protocol, they are named as $2 \mathrm{~S}-\mathrm{XOR}$, 2S-SUP and 2S-SuX.

\section{ANALYSIS OF RATE REGIONS IN GAUSSIAN CHANNELS}

Let $R_{0}$ and $R_{2}$ denote the data rates of the information flows $0 \rightarrow 2$ and $2 \rightarrow 0$, respectively, in the considered twoway communications. In this section, we derive the rate region $\left(R_{0}, R_{2}\right)$ of the aforementioned relay strategies. To the best of our knowledge, the achievable rate regions of bit-level XOR schemes are not reported in the literature and are a part of our contributions. Without loss of generality, we assume $\gamma_{10} \geq \gamma_{12}$ and thus $C_{10} \geq C_{12}$ in Subsections IV-A and B.

\section{A. Achievable rate region for superimposed $X O R$}

Theorem $1(4 S-S u X)$ : The rate region for $4 \mathrm{~S}-\mathrm{SuX}$ is the closure of the set of all rate pairs $\left(R_{0}, R_{2}\right)$ satisfying

$$
\begin{aligned}
\left(R_{0}, R_{2}\right):\left\{R_{0} \leq \min \left(\left(\lambda_{1}+\lambda_{3}\right) C_{01}, \lambda_{1} C_{02}+\lambda_{4} C_{12}(\theta)\right)\right. & , \\
& R_{2} \leq \min \left(\left(\lambda_{2}+\lambda_{3}\right) C_{21}, \lambda_{2} C_{20}+\lambda_{4} C_{10}\right) \\
& R_{2}-R_{0} \leq-\lambda_{1} C_{02}+\lambda_{2} C_{20}+\lambda_{4} C_{10}(1-\theta) \\
& R_{0}+R_{2} \leq \lambda_{1} C_{01}+\lambda_{2} C_{21}+\lambda_{3} C_{\mathrm{m}} \\
& \left.\sum_{i=1}^{4} \lambda_{i}=1, \theta \in[0,1]\right\}
\end{aligned}
$$

Proof: Let $D_{0}$ denote the information packet to be transmitted from node 0 to 2 and its packet length in bits be denoted as $\left|D_{0}\right|$. Assume the message in the packet is further split into two parts, denoted as $D_{0}^{(1)}$ and $D_{0}^{(3)}$, which are transmitted in the first and third step, respectively. Likewise, we let $D_{2}$ denote the information packet to be transmitted from node 2 to 0 , and let it be split into $D_{2}^{(2)}$ and $D_{2}^{(3)}$ for transmission in the second and third steps. During the first three steps of packet transmission (i.e. the multiple-access phase), it is obvious that $\left|D_{0}^{(1)}\right| \leq \lambda_{1} C_{01},\left|D_{2}^{(2)}\right| \leq \lambda_{2} C_{21}$, $\left|D_{0}^{(3)}\right| \leq \lambda_{3} C_{01},\left|D_{2}^{(3)}\right| \leq \lambda_{3} C_{21}$, and $\left|D_{0}^{(3)}\right|+\left|D_{2}^{(3)}\right| \leq$ $\lambda_{3} C_{\mathrm{m}}$. Note that in the first step, due to the presence of direct link between node 0 and node 2 , the desired destination node 2 is able to exact $\left|D_{02}\right| \leq \lambda_{1} C_{02}$ amount of information. Thus, the total amount of information bits to be transmitted through the relay link in the fourth step to node 2 is $\left|D_{0}\right|-\left|D_{02}\right|$ and we denote the corresponding packet as $D_{0}^{\prime}$. Similarly, the total amount of information to be relayed from node 2 to node 0 in the fourth step is $\left|D_{2}\right|-\left|D_{20}\right|$, with $\left|D_{20}\right| \leq \lambda_{2} C_{20}$, and the corresponding packet can be denoted as $D_{2}^{\prime}$. Then, during the fourth step transmission (i.e. the broadcast phase), by comparing the packet sizes $\left|D_{0}^{\prime}\right|$ and $\left|D_{2}^{\prime}\right|$, two cases need to be considered.
Case 1: $\left|D_{2}^{\prime}\right| \geq\left|D_{0}^{\prime}\right|$. The relay node 1 partitions the packet $D_{2}^{\prime}$ into $D_{2}^{\prime(1)}$ and $D_{2}^{\prime(2)}$ so that $\left|D_{2}^{\prime(1)}\right|=\left|D_{0}^{\prime}\right|$ and $\left|D_{2}^{\prime(2)}\right|=$ $\left|D_{2}^{\prime}\right|-\left|D_{0}^{\prime}\right|$. Packet $D_{2}^{\prime(1)}$ contains the first $\left|D_{0}^{\prime}\right|$ bits from $D_{2}^{\prime}$ and packet $D_{2}^{\prime(2)}$ contains the rest of the bits from $D_{2}^{\prime}$. Now, node 1 creates $D_{1}^{\prime}=D_{2}^{\prime(1)} \oplus D_{0}^{\prime}$. Then, the information bits $D_{1}^{\prime}$ and $D_{2}^{\prime(2)}$ are encoded separately into two codewords $\mathbf{x}_{0}^{\prime}$ and $\mathbf{x}_{2}^{\prime}$ with the same length, which are then superimposed together in the complex field. Unlike XOR-based scheme, there is no extra time used to transmit $D_{2}^{\prime(2)}$ to node 0 . Let $\theta$ present the power ratio allocated to the signal $\mathrm{x}_{0}^{\prime}$ to be transmitted to nodes 0 and 2 and $1-\theta$ be the power ratio on the signal $\mathbf{x}_{2}^{\prime}$ to node 0 , where $0 \leq \theta \leq 1$. Due to the assumption that $\gamma_{10} \geq \gamma_{12}$ and the constraint that both nodes 0 and 2 need to receive it successfully, the packet $D_{1}^{\prime}$ is transmitted at the lower rate $C_{12}(\theta)=C\left(\theta \gamma_{12}\right)$ for a fraction $\lambda_{4}$ of time. Thus, we have $\left|D_{0}\right|-\left|D_{02}\right|=\left|D_{0}^{\prime}\right|=\left|D_{1}^{\prime}\right| \leq \lambda_{4} C_{12}(\theta)$. Since node 0 does not know the packets $D_{2}^{\prime(1)}$ and $D_{2}^{\prime(2)}$ and only node 0 needs to decode $\mathbf{x}_{2}^{\prime}$, the link $1 \rightarrow 0$, can be regarded as a virtual MAC and the channel capacity is bound by $\left|D_{2}^{\prime(1)}\right|=$ $\left|D_{1}^{\prime}\right| \leq \lambda_{4} C_{12}(\theta),\left|D_{2}^{\prime}\right|-\left|D_{0}^{\prime}\right|=\left|D_{2}^{\prime}\right|-\left|D_{2}^{\prime(1)}\right|=\left|D_{2}^{\prime(2)}\right| \leq$ $\lambda_{4} C_{10}(1-\theta)$ and $\left|D_{2}^{\prime}\right|=\left|D_{2}^{\prime(1)}\right|+\left|D_{2}^{\prime(2)}\right| \leq \lambda_{4} C\left(\theta \gamma_{10}+\right.$ $\left.(1-\theta) \gamma_{10}\right)=\lambda_{4} C_{10}$. Here $C_{10}(1-\theta)=C\left((1-\theta) \gamma_{10}\right)$. After receiving $\mathbf{x}_{1}=\sqrt{\theta} \mathbf{x}_{0}^{\prime}+\sqrt{1-\theta} \mathbf{x}_{2}^{\prime}$, node 2 extracts the symbol $\mathbf{x}_{0}^{\prime}$ as $\mathbf{x}_{0}^{\prime}=\mathbf{x}_{1}-\sqrt{1-\theta} \mathbf{x}_{2}^{\prime}$, where $\mathbf{x}_{2}^{\prime}$ is encoded by $D_{2}^{\prime(2)}$ which already has been known. By decoding $\mathrm{x}_{0}^{\prime}$, we get $D_{1}^{\prime}$. Then, node 2 extracts the packet $D_{0}^{\prime}$ as $D_{0}^{\prime}=D_{1}^{\prime} \oplus D_{2}^{\prime(1)}$. Similarly, after receiving $\mathbf{x}_{1}$, node 0 extracts $\mathrm{x}_{0}^{\prime}$ and $\mathrm{x}_{2}^{\prime}$ by fully decoding. Then, $D_{1}^{\prime}$ and $D_{2}^{\prime(2)}$ can be obtained easily. Next, node 0 extracts the packet $D_{2}^{\prime(1)}$ as $D_{2}^{\prime(1)}=D_{1}^{\prime} \oplus D_{0}^{\prime}$. Lastly, we have $\sum_{i=1}^{4} \lambda_{i}=1$ due to the total time constraint. Note that, for each destination, say node 2 , to recover the desired packet $D_{0}$ from $D_{02}$ and $D_{0}^{\prime}$, a coding method for Gaussian parallel channel should be employed [16].

With the constraints obtained from the above discussion and using the definition that $R_{0}=\left|D_{0}\right|$ and $R_{2}=\left|D_{2}\right|$ we obtain the set of linear inequalities about $R_{0}$ and $R_{2}$ after simple manipulation for this case:

$$
\begin{aligned}
R_{0} & \leq \min \left(\left(\lambda_{1}+\lambda_{3}\right) C_{01}, \lambda_{1} C_{02}+\lambda_{4} C_{12}(\theta)\right) \\
R_{2} & \leq \min \left(\left(\lambda_{2}+\lambda_{3}\right) C_{21}, \lambda_{2} C_{20}+\lambda_{4} C_{10}\right) \\
R_{2}-R_{0} & \leq-\lambda_{1} C_{02}+\lambda_{2} C_{20}+\lambda_{4} C_{10}(1-\theta) \\
R_{0}+R_{2} & \leq \lambda_{1} C_{01}+\lambda_{2} C_{21}+\lambda_{3} C_{\mathrm{m}} \\
R_{0}-R_{2} & \leq \lambda_{1} C_{02}-\lambda_{2} C_{20}
\end{aligned}
$$

In addition, due to the total time constraint, we have $\sum_{i=1}^{4} \lambda_{i}=1$. Lastly, the power ratio $\theta$ can take any value that satisfies $0 \leq \theta \leq 1$.

Case 2: $\left|D_{2}^{\prime}\right| \leq\left|D_{0}^{\prime}\right|$. The packet $D_{2}^{\prime}$ is padded with zeros to obtain the packet $D_{2}^{\prime p}$ such that $\left|D_{2}^{\prime p}\right|=\left|D_{0}^{\prime}\right|$. Since node 0 and 2 know the size of $D_{2}^{\prime}$, they also know how many zeros are used for padding. Node 1 creates the packet $D_{1}^{\prime}=D_{2}^{\prime p} \oplus D_{0}^{\prime}$. In Step 4 the packet $D_{1}^{\prime}$ is broadcast at a rate at which both node 0 and node 2 can successfully decode. Thus, we have $R_{0}-R_{02}=\left|D_{0}\right|-\left|D_{02}\right|=\left|D_{0}^{\prime}\right|=\left|D_{1}^{\prime}\right| \leq \lambda_{4} C_{12}$. Node 2 then extracts $D_{0}^{\prime}$ as $D_{0}^{\prime}=D_{2}^{\prime p} \oplus D_{1}^{\prime}$, which is the desired 
packet sent from node 0 . Similarly, node 0 can obtain $D_{2}^{\prime p}$ from $D_{1}^{\prime}$. The packet $D_{2}^{\prime}$ is then obtained by removing the padding zeros from $D_{2}^{\prime p}$.

Thus, in this case, we obtain the following linear inequalities that the rate pair $\left(R_{0}, R_{2}\right)$ has to satisfy:

$$
\begin{aligned}
R_{0} & \leq \min \left(\left(\lambda_{1}+\lambda_{3}\right) C_{01}, \lambda_{1} C_{02}+\lambda_{4} C_{12}\right), \\
R_{2} & \leq\left(\lambda_{2}+\lambda_{3}\right) C_{21} \\
R_{0}+R_{2} & \leq \lambda_{1} C_{01}+\lambda_{2} C_{21}+\lambda_{3} C_{\mathrm{m}} \\
R_{2}-R_{0} & \leq-\lambda_{1} C_{02}+\lambda_{2} C_{20}
\end{aligned}
$$

Finally, combining the set of results for case 2 with the results for case 1 , we obtain the rate region of $4 \mathrm{~S}-\mathrm{SuX}$ as given in the theorem.

Remark: Let $\lambda_{1}=\lambda_{2}=0$ in Theorem 1, the rate region for $2 \mathrm{~S}-\mathrm{SuX}$ is obtained.

Theorem $2(2 \mathrm{~S}-\mathrm{SuX})$ : The rate region for $2 \mathrm{~S}-\mathrm{SuX}$ is the closure of the set of all rate pairs $\left(R_{0}, R_{2}\right)$ satisfying

$$
\begin{aligned}
& \left(R_{0}, R_{2}\right):\left\{R_{0} \leq \min \left(\lambda_{1} C_{01}, \lambda_{2} C_{12}(\theta)\right),\right. \\
& R_{2} \leq \min \left(\lambda_{1} C_{21}, \lambda_{2} C_{10}\right), \\
& R_{2}-R_{0} \leq \lambda_{2} C_{10}(1-\theta), \\
& R_{0}+R_{2} \leq \lambda_{1} C_{\mathrm{m}}, \\
& \left.\sum_{i=1}^{2} \lambda_{i}=1, \theta \in[0,1]\right\} .
\end{aligned}
$$

\section{B. Achievable rate region for $X O R$}

Theorem 3 (4S-XOR): The rate region for $4 \mathrm{~S}-\mathrm{XOR}$ is the closure of the set of all rate pair $\left(R_{0}, R_{2}\right)$ satisfying

$$
\begin{aligned}
& \left(R_{0}, R_{2}\right):\left\{R_{0} \leq \min \left(\left(\lambda_{1}+\lambda_{3}\right) C_{01}, \lambda_{1} C_{02}+\lambda_{4} C_{12}\right),\right. \\
& R_{0}+R_{2} \leq \lambda_{1} C_{01}+\lambda_{2} C_{21}+\lambda_{3} C_{\mathrm{m}}, R_{2} \leq\left(\lambda_{2}+\lambda_{3}\right) C_{21}, \\
& \left.R_{2}-R_{0} \leq-\lambda_{1} C_{02}+\lambda_{2} C_{20}+\lambda_{5} C_{10}, \sum_{i=1}^{5} \lambda_{i}=1\right\} .
\end{aligned}
$$

Proof: The proof of this theorem differs from the proof of Theorem 1 mainly in Case 1 of the broadcast phase. The coding and decoding method of Case 1 are similar to those discussed in [12], [13] and are omitted.

Remark: Let $\lambda_{1}=\lambda_{2}=0$ in Theorem 3, we obtain the rate region for $2 \mathrm{~S}-\mathrm{XOR}$.

Theorem 4 (2S-XOR): The rate region for $2 \mathrm{~S}$-XOR is the closure of the set of all rate pair $\left(R_{0}, R_{2}\right)$ satisfying

$$
\begin{aligned}
& \left(R_{0}, R_{2}\right):\left\{R_{0} \leq \min \left(\lambda_{1} C_{01}, \lambda_{2} C_{12}\right), R_{2} \leq \lambda_{1} C_{21},\right. \\
& \left.R_{0}+R_{2} \leq \lambda_{1} C_{\mathrm{m}}, R_{2}-R_{0} \leq \lambda_{3} C_{10}, \sum_{i=1}^{3} \lambda_{i}=1\right\} .
\end{aligned}
$$

\section{Achievable rate region for superposition coding}

The achievable rate region of 2S-SUP is well studied in [7], [14], [17]. Though the achieve rate region of 4S-SUP is not studied yet in the literature, its derivation is trivial.

Theorem $5(4 S-S U P)$ : The rate region for $4 \mathrm{~S}-\mathrm{SUP}$ is the closure of the set of all rate pair $\left(R_{0}, R_{2}\right)$ satisfying

$$
\begin{aligned}
& \left(R_{0}, R_{2}\right):\left\{R_{0} \leq \min \left(\left(\lambda_{1}+\lambda_{3}\right) C_{01}, \lambda_{1} C_{02}+\lambda_{4} C_{12}(\theta)\right),\right. \\
& R_{2} \leq \min \left(\left(\lambda_{2}+\lambda_{3}\right) C_{21}, \lambda_{2} C_{20}+\lambda_{4} C_{10}(1-\theta)\right), \\
& \left.R_{0}+R_{2} \leq \lambda_{1} C_{01}+\lambda_{2} C_{21}+\lambda_{3} C_{\mathrm{m}}, \sum_{i=1}^{4} \lambda_{i}=1, \theta \in[0,1]\right\}
\end{aligned}
$$

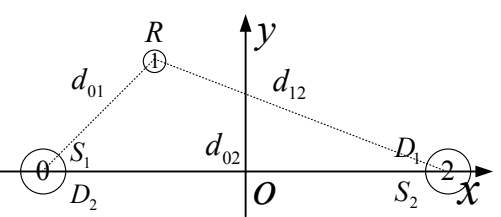

Fig. 1: Layout of the two-way relaying network

Theorem $6(2 S-S U P)$ : The rate region for $2 \mathrm{~S}-\mathrm{SUP}$ is the closure of the set of all rate pair $\left(R_{0}, R_{2}\right)$ satisfying

$$
\begin{aligned}
& \left(R_{0}, R_{2}\right):\left\{R_{0} \leq \min \left(\lambda_{1} C_{01}, \lambda_{2} C_{12}(\theta)\right), R_{0}+R_{2} \leq \lambda_{1} C_{\mathrm{m}},\right. \\
& \left.R_{2} \leq \min \left(\lambda_{1} C_{21}, \lambda_{2} C_{10}(1-\theta)\right), \sum_{i=1}^{2} \lambda_{i}=1, \theta \in[0,1]\right\} .
\end{aligned}
$$

\section{Cases with direct transmission}

For 4-step strategies, it is assumed by default that the direct link is always worse than the relay link. That is, $C_{02}<C_{01}$ and $C_{20}<C_{21}$. In this subsection, we consider the special cases where the direct link is better.

If $C_{02}<C_{01}$ and $C_{20} \geq C_{21}$, the signal from node 2 will be transmitted directly to node 0 without the help of relay. This corresponds to $\lambda_{3}=0$ and the relay transmitting to node 2 only during $\lambda_{4}$ in the 4-step protocol. Thus, using Theorem 1 , we obtain the sets of rate pairs $\left(R_{0}, R_{2}\right)$ satisfying

$$
\begin{aligned}
& \left(R_{0}, R_{2}\right):\left\{R_{0} \leq \min \left(\lambda_{1} C_{01}, \lambda_{1} C_{02}+\lambda_{2} C_{12}\right),\right. \\
& \left.R_{2} \leq \lambda_{3} C_{20}, \sum_{i=1}^{3} \lambda_{i}=1\right\} .
\end{aligned}
$$

If $C_{02} \geq C_{01}$ and $C_{20}<C_{21}$, the signal from node 0 will be transmitted directly to node 2 without the help of relay. The sets of rate pairs $\left(R_{0}, R_{2}\right)$ satisfy

$$
\begin{aligned}
& \left(R_{0}, R_{2}\right):\left\{R_{2} \leq \min \left(\lambda_{2} C_{21}, \lambda_{2} C_{20}+\lambda_{3} C_{10}\right),\right. \\
& \left.R_{0} \leq \lambda_{1} C_{01}, \sum_{i=1}^{3} \lambda_{i}=1\right\} .
\end{aligned}
$$

If $C_{02} \geq C_{01}$ and $C_{20} \geq C_{21}$, direct communication between node 0 and node 2 in both ways is preferred and no relay is needed. Hence, the rate pairs are given by

$$
\left(R_{0}, R_{2}\right):\left\{R_{0} \leq \lambda_{1} C_{02}, R_{2} \leq \lambda_{2} C_{20}, \sum_{i=1}^{2} \lambda_{i}=1\right\} .
$$

\section{E. Some numeral results}

Suppose that the channel gain on each link is modeled by the distance path loss model, given by $\alpha_{i j}=c \cdot d_{i j}^{-n}$, where $c$ is an attenuation constant, $n$ is the path loss exponent and fixed at 3 , and $d_{i j}$ denotes the distance between nodes $i$ and $j$. For simplicity, each node uses the same transmission power $P$, though our analytical results are suitable for the case with unequal transmit power. The noise power is assumed to one. We consider the network layout shown in Fig. 1, where the distance between nodes 0 and 2 is normalized to 1 and the location of the relay is determined using the projections $x$ and $y$. The source nodes 0 and 2 are located at coordinates $(-0.5,0)$ and $(0.5,0)$, respectively. The distances from the relay to the source nodes can be computed as $d_{01}=\sqrt{(x+0.5)^{2}+y^{2}}$, and $d_{12}=\sqrt{(x-0.5)^{2}+y^{2}}$.

Figs. 2-4 illustrate the rate regions of different two-way relay strategies. For comparison, the achievable rate region of 


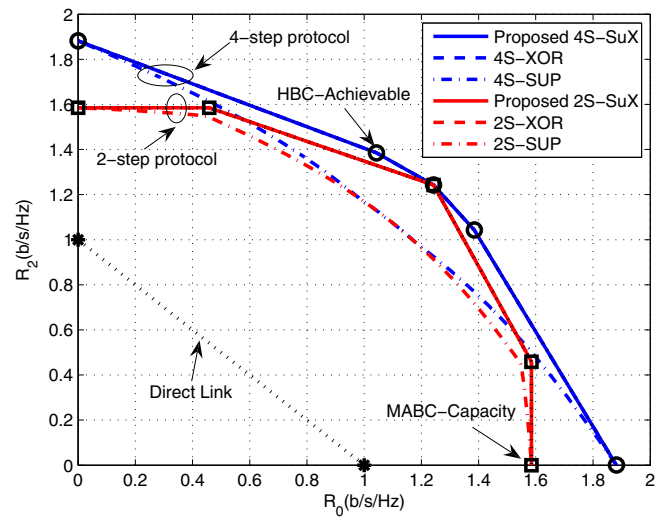

Fig. 2: Rate region at $(x, y)=(0,0)$ with $P=0 \mathrm{~dB}$.

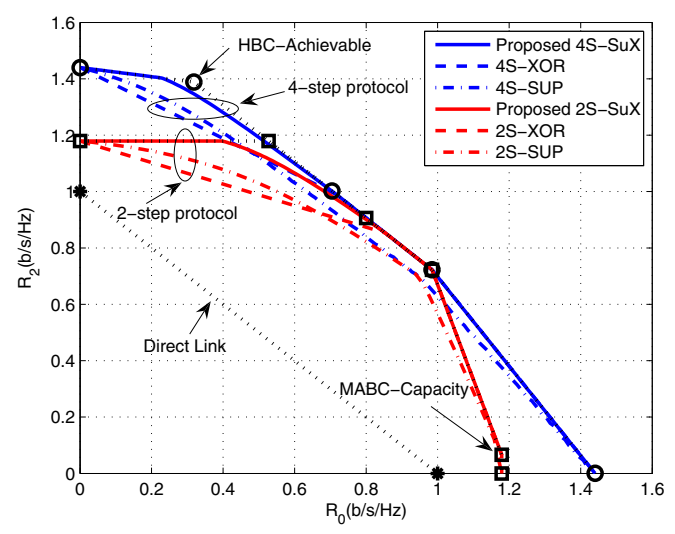

Fig. 3: Rate region at $(x, y)=(-0.2,0.3)$ with $P=0 \mathrm{~dB}$.

the HBC protocol (4-step protocol) [10] and the capacity of the MABC protocol (2-step protocol) derived in [8]-[10] are also shown and denoted by marks only in the figures. From Fig. 2, where the two relay channels are symmetrical, we see that the SuX and XOR schemes are capacity achieving whereas the SUP scheme is much inferior.

From Figs. 3-4, where the two relay channels are asymmetrical, it is observed that XOR is far from capacity-achieving and that SUP schemes becomes better than XOR schemes if $R_{2}>R_{0}$. On the other hand, the proposed SuX schemes closely approach the capacity (or the best achievable rate region) obtained by random binning in the high SNR regime (Fig. 4), while there is only a minor gap in the low SNR regime (Fig. 3).

\section{Performance Comparison in Fading Channels}

In this section, we present a numerical study of the proposed superimposed XOR in terms of two performance metrics over fading channels: system average sum-rate and service delay performance. The same network layout and channel model as described in Section IV-E are used, except that smallscale fading is included here. We assume the fading on each link follows Rayleigh distribution and are independent and reciprocal for different links.

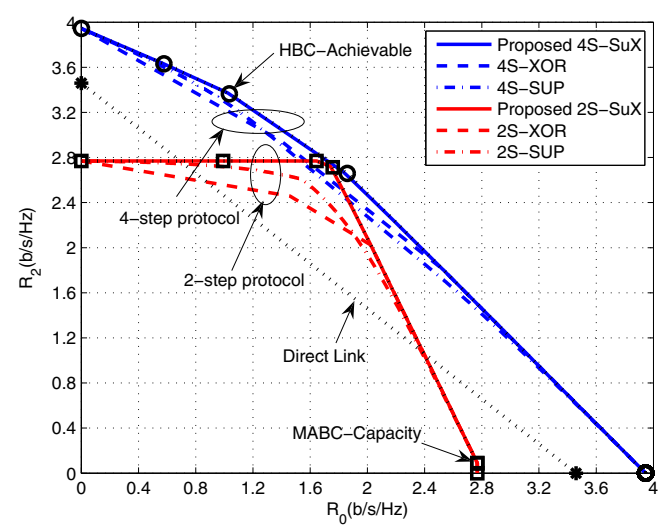

Fig. 4: Rate region at $(x, y)=(-0.2,0.3)$ with $P=10 \mathrm{~dB}$.

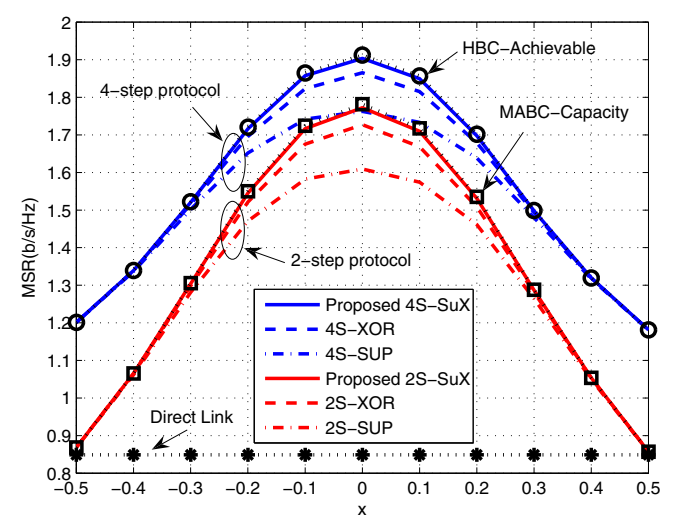

Fig. 5: Averaged maximum sum-rates versus relay location when $-0.5 \leq x \leq 0.5, y=0$ with $P=0 \mathrm{~dB}$.

\section{A. Maximum sum-rates (MSRs)}

Firstly, we consider the maximum sum-rate. The problem is formulated as

$$
\max _{\left(R_{0}(t), R_{2}(t)\right) \in \mathcal{R}(t)} R_{0}(t)+R_{2}(t)
$$

where $R_{k}(t), k \in\{0,2\}$, denotes the service rate of node $k$ at the $t$-th time slot, and $\mathcal{R}(t)$ stands for the rate region with respect to the channel realization at the $t$-th time slot.

Figs. 5-6 show the averaged MSRs of different two-way relay strategies, when the relay node moves alone the line between the two source nodes. It is observed that no matter where the relay is, the proposed $\mathrm{SuX}$ scheme always achieves the largest sum-rates among all the considered PLNC schemes (XOR, SUP and SuX) and approach the optimal bounds very well, especially for high SNR. We can also see as the relay node is approaching one source node (i.e. $x$ approaches 0.5 or -0.5$)$, the performances of the considered PLNC schemes converge.

\section{B. Service delay}

Here, we use the same queuing mode as in [17], in which service rate allocation is done by a cross-layer approach by taking into account both queue length and channel state. Note that, Oechtering, et al. in [17] only focus on 2S-SUP strategies 


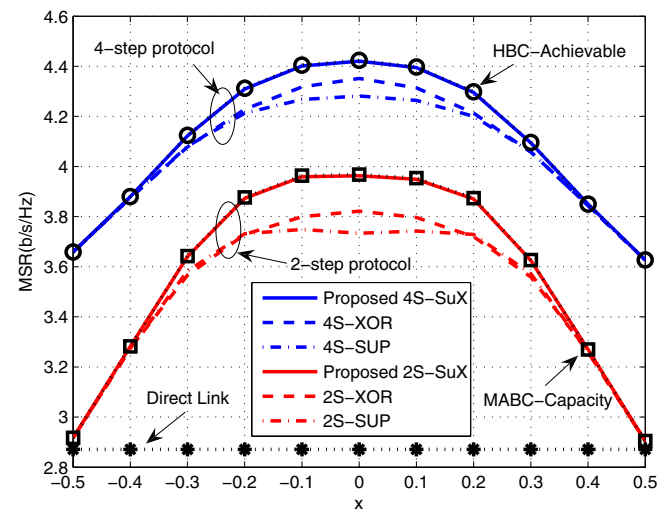

Fig. 6: Averaged maximum sum-rates versus relay location when $-0.5 \leq x \leq 0.5, y=0$ with $P=10 \mathrm{~dB}$.

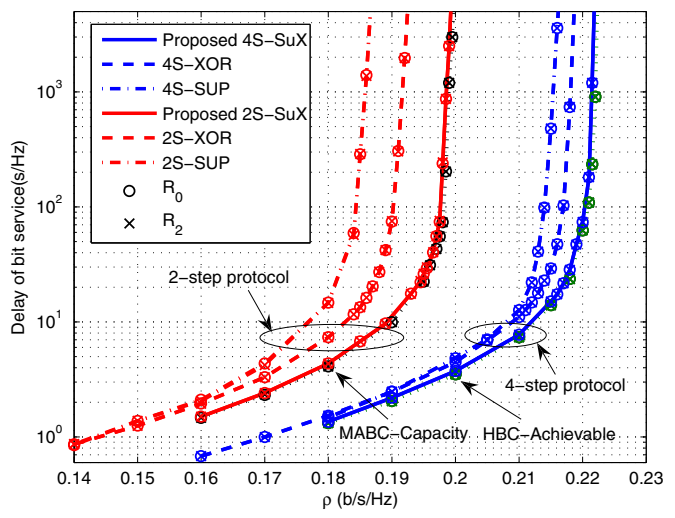

Fig. 7: System service delay versus packet arrival rate at $(x, y)=(0,0)$ with $P=10 \mathrm{~dB}$.

and consider the queue backlog versus bit arrival rate. Chen, et al. considered delay power tradeoff in [18], and assumed all the links have the same rate and only the relay has buffer. We study the bit delay versus packet arrival rate. Suppose that the packet arrival at two source nodes follow Poisson distribution with mean $\rho$, the length of each packet is fixed as $L$ bits. Let $Q(t-1)=\left[Q_{0}(t-1), Q_{2}(t-1)\right]$ represent the remaining bits in the queues after the $(t-1)$-th time slot. Then, $Q(t)=\left[Q_{0}(t), Q_{2}(t)\right]=\left[Q_{0}(t-1)-R_{0}(t)+A_{0}(t) L, Q_{2}(t-\right.$ $\left.1)-R_{2}(t)+A_{2}(t) L\right]$, where $R_{k}(t), A_{k}(t)(k \in\{0,2\})$ denote the service rates and packet arrival rates of node $k$ in $t$-th time slot. The rate allocation problem is formulated as

$$
\max _{\substack{\left(R_{0}(t), R_{2}(t)\right) \in \mathcal{R}(t) \\ R_{0}(t) \leq Q_{0}(t-1), R_{2}(t) \leq Q_{2}(t-1)}} Q_{0}(t-1) R_{0}(t)+Q_{2}(t-1) R_{2}(t)
$$

Fig. 7 shows the system delay based on the above weighted sum-rate maximization with $L=10$. It can be clearly seen that the proposed SuX scheme always outperforms the other two PLNC schemes, no matter which time control protocol is applied.

\section{CONCLUSION}

In this paper, we proposed a new practical PLNC scheme called superimposed XOR, or SuX, for two-way relay com- munications. Combined with two transmission protocols, we characterized the corresponding achievable rate regions over Gaussian channels and demonstrate its average maximum sumrate and service delay performance over fading channels. For comparison, the traditional bitwise XOR and symbol-level superposition PLNC schemes were also considered. Numerical results showed that the proposed SuX scheme always outperforms the XOR and superposition based PLNC schemes when the relay channels are asymmetrical. Compared with the ideal random binning approach, the proposed practical PLNC scheme SuX approaches the capacity very well, especially in the high SNR regime.

\section{REFERENCES}

[1] Y. Wu, P. A. Chou, and S.-Y. Kung, "Information exchange in wireless networks with network coding and physical-layer broadcast," in Proc. 39th Conf. on Inform. Sciences and Systems (CISS), Mar. 2005.

[2] P. Larsson, N. Johansson, and K.-E. Sunell, "Coded bi-directional relaying," in Proc. 5th Scandinavian Workshop on Ad Hoc Networks (ADHOC), May 2005.

[3] P. Popovski and H. Yomo, "Bi-directional amplification of throughput in a wireless multi-hop network," in Proc. IEEE Veh. Tech. Conf. (VTC), May 2006, pp. 588-593.

[4] C. Hausl and J. Hagenauer, "Iterative network and channel decoding for the two-way relay channel," in Proc. IEEE Int. Conf. on Comm. (ICC), Jun. 2006, pp. 1568-1573.

[5] S. Zhang, S. C. Liew, and P. P. Lam, "Physical-layer network coding," in Proc. ACM MobiCom, Sept. 2006, pp. 358-365.

[6] S. Katti, H. Rahul, W. Hu, D. Katabi, M. M. edard, and J. Crowcroft, "XORs in the air: Practical wireless network coding," in Proc. ACM SIGCOMM, Sept. 2006, pp. 243-254.

[7] B. Rankov and A. Wittneben, "Spectral efficient protocols for halfduplex fading relay channels," IEEE Jour. Selec. Areas. Comm., vol. 25, no. 2, pp. 379-389, Feb. 2007.

[8] T. J. Oechtering, C. Schnurr, I. Bjelakovic, and H. Boche, "Broadcast capacity region of two-phase bidirectional relaying," IEEE Trans. Inform. Theory, vol. 54, no. 1, pp. 454-458, Jan. 2008.

[9] L. L. Xie, "Network coding and random binning for multi-user channels," in Proc. IEEE 10th Canadian Workshop on Inform. Theory, June 2007, pp. 85-88.

[10] S. J. Kim, P. Mitran, and V. Tarokh, "Performance bounds for bidirectional coded cooperation protocols," IEEE Trans. Inf. Theory, vol. 54, no. 11, pp. 5235-5241, Nov. 2008.

[11] S. J. Kim, N. Devroye, P. Mitran, and V. Tarokh, "Comparison of bidirectional relaying protocols," in IEEE Sarnoff Symposium, April 2008, pp. $1-5$.

[12] P. Popovski and H. Yomo, "Physical network coding in two-way wireless relay channels," in Proc. IEEE Int. Conf. on Comm. (ICC), June 2007, pp. $707-712$.

[13] C. H. Liu and F. Xue, "Network coding for two-way relaying: rate region, sum rate and opportunistic scheduling," in Proc. IEEE Int. Conf. on Comm. (ICC), May 2008, pp. 1044-1049.

[14] T. J. Oechtering and H. Boche, "Optimal time-division for bi-directional relaying using superposition encoding," IEEE Coтmu. Letters, vol. 12, no. 4, pp. 265-267, April 2008.

[15] C. H. Liu and A. Arapostathis, "Joint network coding and superposition coding for multi-user information exchange in wireless relaying networks," in Proc. IEEE Global Telecomm. Conf. (GLOBECOM), Nov. 2008, pp. 1-6.

[16] A. H.-Madsen, "Capacity bounds for cooperative diversity," IEEE Trans. Inform. Theory, vol. 52, no. 4, pp. 1522-1544, April 2006.

[17] T. J. Oechtering and H. Boche, "Stability region of an optimized bidirectional regenerative half-duplex relaying protocol," IEEE Trans. Сотти., vol. 56, no. 9, pp. 1519-1529, Sep. 2008.

[18] W. Chen, K. B. Letaief, and Z. Cao, "Opportunistic network coding for wireless networks," in Proc. IEEE Int. Conf. on Comm. (ICC), 2007, pp. 4634-4639. 